\title{
HIGH CURRENT DENSITY NEGATIVE ION SOURCE FOR BEAM LINE TRANSPORT STUDIES
}

\author{
Vadim Dudnikov, Charles W. Schmidt, Ray Hren and James Wendt \\ Fermi National Accelerator Laboratory*, Batavia, IL 60510, USA
}

\begin{abstract}
The Fermilab Electron Cooling Program requires a 20$\mathrm{m}$ solenoidal region to interact $8-\mathrm{GeV}$ antiprotons with an escorting beam of $4.3-\mathrm{MeV}$ electrons to improve the phase-space quality of the antiproton beam. The solenoidal section with additional transport lines to take and return a 0.5 -A electron beam from an electrostatic accelerator, for energy recovery, must be precisely aligned and adjusted. For the initial setup and study, and later testing of this line, a $12.4-\mathrm{keV} \mathrm{H}^{-}$beam can be used to simulate the $4.3 \mathrm{MeV}$ electron beam. For this purpose a high-brightness $\mathrm{H}^{-}$ion source has been developed and tested. The source, a semiplanatron type, with a hollow cathode discharge and spherical cathode focusing of the emitted ions to the emission aperture has given an emission current density up to $0.7 \mathrm{~A} / \mathrm{cm}^{2}$. Continuous operation of 4 weeks has been demonstrated. Such an optimized source could have many applications for tandem accelerators, ion beam lithography and ion implantation.
\end{abstract}

\section{INTRODUCTION}

For efficient cooling of antiprotons an escorting electron beam should have very low angular spread, $\Delta \alpha<0.08 \mathrm{mrad}$ [1]. The beam line optic should have low aberrations to suppress any electron beam heating. To detect the low level of aberration an ion beam with a small emittance and high brightness is needed, as for ion lithography [2]. $\mathrm{H}^{-}$beams with high brightness have been produced from pulsed Surface Plasma Sources (SPS) with a charge-exchange conversion giving cooling to the ions [3]. For DC production of a negative ion beam with a high emission current density, a SPS with a hollow cathode discharge and spherical geometrical focusing of the negative ions was developed [4]. An $\mathrm{H}^{-}$beam with an intensity $2.5 \mathrm{~mA}$ has been extracted from a $1 \mathrm{~mm}$-diameter aperture. The corresponding emission current density is $0.3 \mathrm{~A} / \mathrm{cm}^{2}$. Some further modifications of this type of SPS were made and tested for use in such beam line optimization.

\section{SPS CONFIGURATION}

For field characterization of the transport bending magnets a small ion beam is used formed by two collimators with a millimeter aperture separated by $3 \mathrm{~m}$. A similar set of apertures detects the exit beam position and angle from the magnet. An ion beam from the source following a solenoidal lens and mass analyzer should pass

\footnotetext{
* Work supported by the U.S. Department of Energy under contract No. DE-AC02-76CH03000. Dudnikov@fnal.gov
}

through a series of $1 \mathrm{~mm}$ apertures over a distance of $8 \mathrm{~m}$ and have an intensity higher than $1 \mathrm{nA}$ for easy detection. More detail of such a test system is discussed in [5]. In early tests a semiplanatron SPS similar to [4], built in cooperation with BTG, was used. A basic configuration of this SPS is shown in Fig.1a. Some modifications are presented in Fig. $1 \boldsymbol{b}$ and $1 \boldsymbol{c}$.
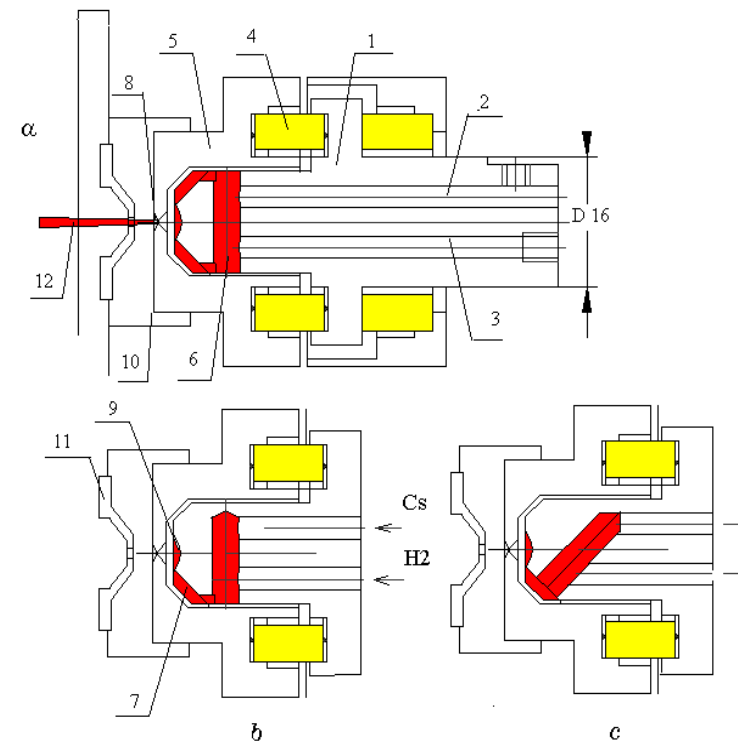

Figure 1. Modifications of the Hollow Cathode Discharge region in a DC SPS. $\boldsymbol{a}$ - perpendicular channel; $\boldsymbol{b}$ - closed perpendicular channel; $\boldsymbol{c}$ - closed 45-degree channel. (discharge plasma is red).

A glow discharge in the magnetic field is supported by a voltage between the cathode (1) and anode (5) separated by a ceramic insulator (4). The diameter of the cylindrical cathode body is $16 \mathrm{~mm}$. The discharge, localized in the cylindrical channel of the hollow cathode (6), is $3 \mathrm{~mm}$ in diameter. The working gas $\left(\mathrm{H}_{2}\right)$ is delivered to the hollow cathode region through channel (3) from the gas system. The gas flow and pressure is controlled by a mechanical leak. Cesium is delivered to the discharge region through channel (2) from a small oven filled with pellets of cesium chromate and titanium powder mixture. Heating evolves a pure cesium vapor from the mixture. The plasma drifts in the crossed field $(\mathrm{ExB})$ along the groove (7) to the spherical surface of the emitter (9). The direction of the plasma drift is determined by the direction of the magnetic field. The emitter's surface (9) is bombarded by positive ions and neutrals from the plasma. Secondary negative ions, emitted from the spherical 
surface (9) are accelerated by the near surface potential well, without space charge limitation, and focused to the extraction aperture (8) with diameter of 0.3 to $1.2 \mathrm{~mm}$. A beam of negative ions (12) is extracted by a high voltage applied between the anode (5) and extractor (11). A magnetic field, formed by permanent magnets, is between two poles (10). During operation this ion source is located in a vacuum chamber so the SPS parts don't need special vacuum sealing. The axial design simplified machining, assembling and maintenance of this SPS. Assembly of this SPS in the vacuum chamber is shown in Fig. 2.

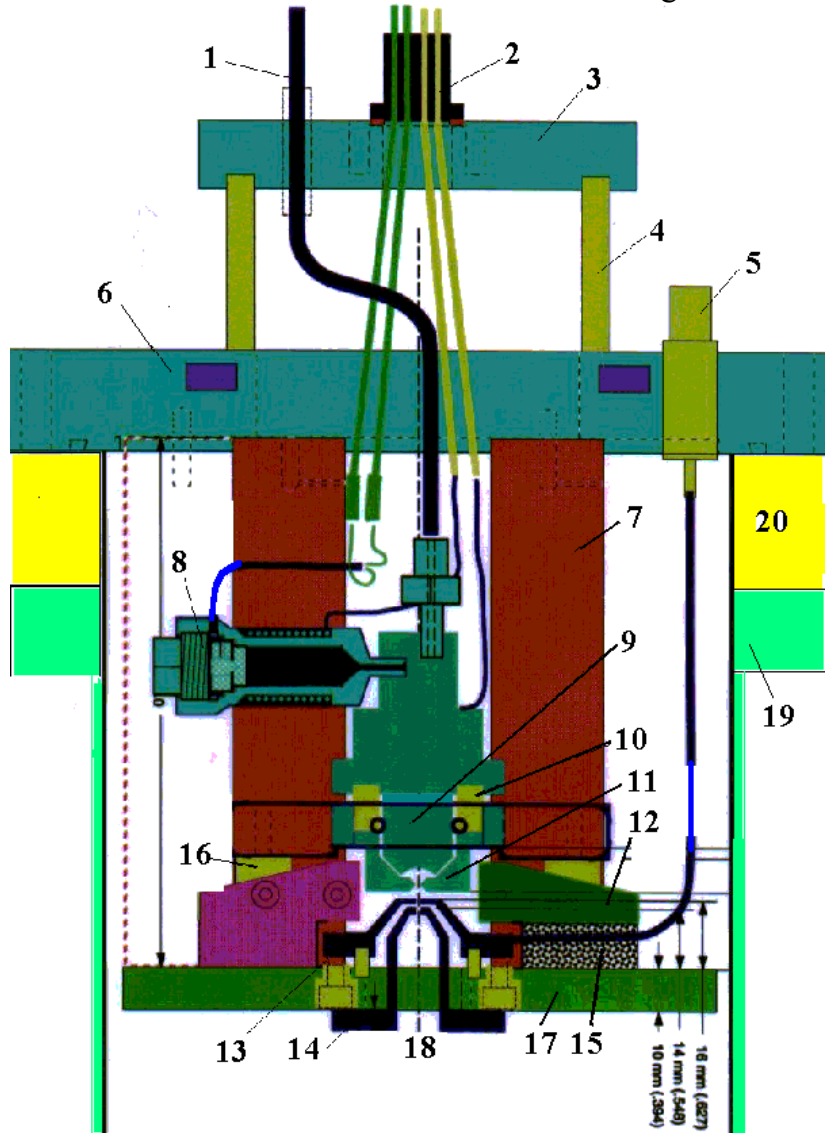

Figure 2. Assembly of the negative ion source in vacuum chamber. 1- gas tube; 2 - electric vacuum feedthroughs; 3high voltage flange; 4- high voltage insulator; 5- high voltage feedthrough; 6- base flange; 7- cooling rods; 8- Cs catalyst supply; 9- cathode-emitter; 10- cathode insulator; 11- gas discharge chamber anode; 12- magnet poles; 13suppression electrode; 14- extraction electrode; 15permanent magnet; 16- high voltage insulators; 17- base plate-magnetic yoke; 18- ion beam; 19- vacuum chamber; 20 - high voltage insulator.

Referring to Figure 2 the base flange, 6, of the ion source supports the entire ion source assembly. In beam line test it mounts to a standard six inch diameter six way vacuum cross, 19, also used for a duoplasmatron proton source. A cylindrical high voltage insulator, 20, for the Duoplasmatron is used for additional biasing of the SPS. Flange, 6 , is cooled by an insulating liquid. Insulator 20 is made from Teflon and Viton O-rings are adequate for vacuum sealing. One flange of the vacuum chamber, 19, is used for ion source attachment. The opposite flange is connected to the beam line. One of the four perpendicular flanges is used for pumping ( $400 \mathrm{l} / \mathrm{s}$ turbopump) while the other is for beam and source diagnostics and for beam control. The base plate of the ion source, 17 , is connected to the mounting plate, 6 , by four copper rods, 7 , which also serve for cooling. The ion source assembly and gas discharge chamber, 11, are attached to the base plate through a high voltage cylindrical ceramic insulator, 16, which is a standard design for the ion implanter industry. In operation, the source anode is biased negative up to -15 $\mathrm{kV}$. The ion emitter-cathode, 9, is separated from the anode, 11, by a ceramic insulator, 10. A compact cesium supply oven, 8 , delivers a catalyst to the emitter surface for enhanced negative ion formation in the gas discharge. The working gas for support of the discharge is delivered from the external gas system through tube 1. For electrical power to the ion source, a multiple feedthrough, 2 , on the high voltage flange, 3 , is used. It is insulated from flange 6 by cylindrical insulator 4 . A three electrode extraction system with a positive suppression electrode, 13, and a grounded extractor, 14, is used for ion extraction and beam formation. The suppression voltage reflects positive ions from the ion beam to improve space charge neutralization and suppress bombardment of the ion source by accelerated positive ions. A magnetic field up to $0.8 \mathrm{kG}$ for the crossed field discharge is formed by the permanent magnets, 15 , poles, 12 , and part of the base plate, 17, which is made from mild steel. The central nonmagnetic platform holding the ion source and extractor can have a small angle to compensate for the ion beam deflection by the magnetic field. A second step of acceleration could be used for beam acceleration above $10-15 \mathrm{keV}$.

\section{TEST RESULTS}

Operation of this SPS was examined in the beam line test. A two or three electrode extraction optics with a suppression electrode was used for beam formation with a solenoidal magnetic lens for final focusing. Beam diagnostic was a moving beam collector for intensity, a luminescent screens of ruby ceramic for source beam observation, and a quartz or YAG single crystal with a CCD camera for fine beam observations. Magnetic deflectors were used to manipulate the beam. An Emittance scanner was used in early tests for emittance measurement. The first SPS was a configuration from Fig.1a: a hollow cathode channel open from on both sides. The focusing spherical radius was $\mathrm{R}=3.5 \mathrm{~mm}$. With only hydrogen gas the discharge voltage was $0.4-0.5 \mathrm{kV}$ so sputtering and flake formation was significant. After adding cesium, the discharge voltage dropped to $100 \mathrm{~V}$ and below. Stable DC operation has been reached with optimized heating of the cesium oven. In the previous tests [4] up to $2.5 \mathrm{~mA}$ of $\mathrm{H}^{-}$was extracted through an extraction aperture of $1 \mathrm{~mm}$ diameter with a discharge voltage of $80 \mathrm{~V}$ and a current of $0.8 \mathrm{~A}$. The emittance $(90 \%)$ of a $25-\mathrm{keV} \mathrm{H}^{-}$beam was $\varepsilon=26 \pi \mathrm{mm} \mathrm{mrad}$. The transverse ion temperature on the extraction surface is 
$\mathrm{T}_{\mathrm{i}} \sim 3 \mathrm{eV}$. An effective brightness, $\mathrm{B}=\mathrm{j}^{\mathrm{j}} / \mathrm{T}_{\mathrm{i}}=0.1 \mathrm{~A} / \mathrm{cm} \mathrm{eV}$, was relative high, but 10 times smaller then for a pulsed SPS [3]. For the beam line test the extraction aperture of 1 $\mathrm{mm}$ diameter was closed to $0.2 \mathrm{~mm}$ stainless steel foil with aperture $0.3 \mathrm{~mm}$. With this aperture the $\mathrm{H}^{-}$current in a remote collector after the solenoid lens, collimator and analyzer was 1 microamp, similar to the proton current from a Duoplasmatron (3 microamp). After 20 hours of operation the collector current started to increase and was 8 microamps after 60 hours. This current growth was due to an aperture increase by sputtering from back accelerated positive ions. With molybdenum foil this sputtering was invisible. In a clean vacuum an increasing current in extractor gap was observed, consisting of electrons and ions, independent of the gas and discharge. This emission was suppressed by a controlled leak of air. With a $0.3 \mathrm{~mm}$ extraction aperture the $\mathrm{H}^{-}$beam was transmitted through the $8 \mathrm{~m}$ long $1 \mathrm{~mm}$ aperture beam line and final intensity was comparable with the proton beam from the Duoplasmatron although repeatability of the $\mathrm{H}^{-}$ beam parameters was not stable. In the $\boldsymbol{a}$ configuration a discharge can start from the top exit of the hollow cathode with a plasma drift to the right, to insulator (4), with a loss of ion emission.

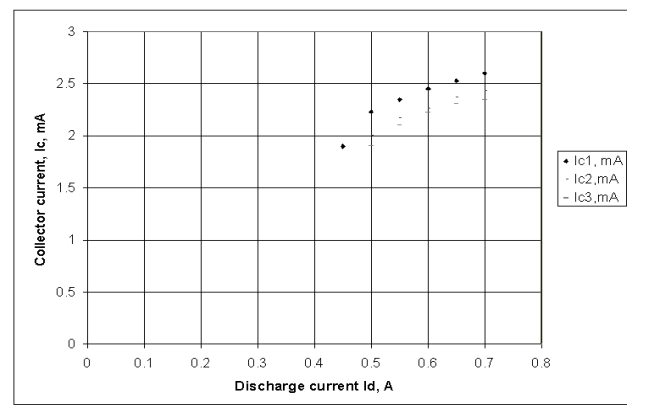

Figure 3: Collector current versus discharge current for SPS b with an extraction aperture of $1 \mathrm{~mm}$ diameter, $\mathrm{Uex}=20 \mathrm{kV}$.

This loss of emission was the reason to change to configuration $\boldsymbol{b}$ with the hollow cathode opening on only one side. This version of the SPS was tested in another test stand with extended operation of up to 4 weeks. This time was limited by the cesium in the oven. For longer operation a larger Cs supply is needed, as in the FNAL magnetron SPS [6]. For long operation is important to have at all times a low discharge voltage $(\mathrm{Ud}<90 \mathrm{~V})$ and good Cs recycling. With an extraction aperture of $1 \mathrm{~mm}$ diameter an extracted $\mathrm{H}^{-}$beam up to $2.5 \mathrm{~mA}$ was repeatedly obtained. Dependence of the beam current at a test stand collector on the discharge current is shown in Figure 3. The collector current saturation is determined not by $\mathrm{H}^{-}$stripping in the plasma, but by an increase of the cathode temperature and increased Cs desorption.

With a new anode and extraction aperture of $0.4 \mathrm{~mm}$ diameter an $\mathrm{H}^{-}$beam up to $0.9 \mathrm{~mA}$ was extracted (emission current density $\mathrm{j}=0.7 \mathrm{~A} / \mathrm{cm}^{2}$ ). The discharge voltage is $\mathrm{Ud}=80 \mathrm{~V}$, discharge current $\mathrm{Id}=0.5 \mathrm{~A}$, power $\mathrm{P}=40 \mathrm{~W}$. The production efficiency at this current density.
$\mathrm{F}=\mathrm{j} / \mathrm{P}=17.5 \mathrm{~A} / \mathrm{cm}^{2} \mathrm{~kW}$, is much higher than $\mathrm{F}=0.25-0.05$ $\mathrm{A} / \mathrm{cm}^{2} \mathrm{~kW}$ for a good proton source [6].

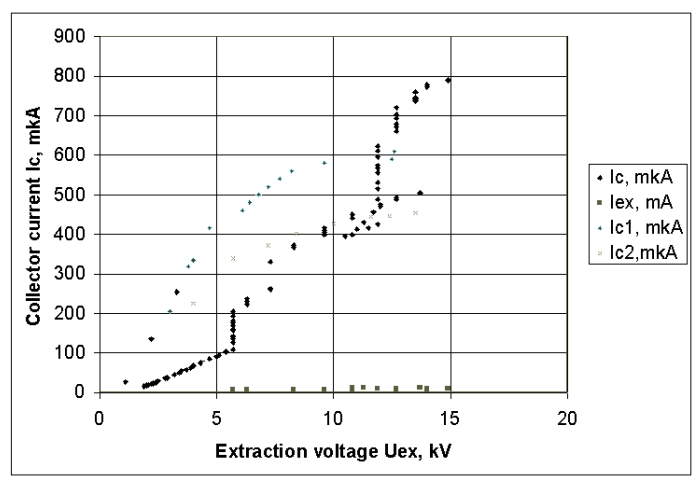

Figure 4: Optimization of the beam current for different extraction voltage for SPS $\mathbf{c}$ with an extraction aperture of $0.4 \mathrm{~mm}$ diameter.

The next improvement is shown in Fig.1 c. A hollow cathode channel was drilled normal to the conical part of the cathode surface and 45 degrees to the axis. This SPS minimized the distance for plasma drift to the emitter surface. The efficiency of negative ion generation has been improved. Examples of the collector current for different condition of SPSc operation are shown in Figure 4. A typical discharge voltage is $\mathrm{Ud}=80 \mathrm{~V}$, discharge current $\mathrm{Id}=0.4 \mathrm{~A}$. Increasing the $\mathrm{H}_{2}$ gas from $\mathrm{p}=10^{-6}$ Torr to $3 \times 10^{-6}$ Torr decreases the collector current from $0.8 \mathrm{~mA}$ to $0.3 \mathrm{~mA}$. Traces of sputtering on the anode surface has conform good focusing of the negative ions by the spherical surface of the emitter. Moving the focusing point relative to the extraction aperture could change the intensity. Conditions for long time operation without any change in the parameters has been found. Divergence of this high current density beam was relative large $\sim 50$ $\mathrm{mrad}$ in agreement with a computer simulation by PBGUN code [8]. With an electrostatic einzel lens after the extractor, the $\mathrm{H}^{-}$beam can be transformed to a converging, parallel or a finely focused on the ruby ceramic screen. This final Semiplanatron SPS could be used for precision optimization of low aberration beam lines and for other applications of high brightness beam.

\section{REFERENCES}

[1] A.Shemyakin, A.Burov, et al., EPAC 2000, Viena, 2000, THU05A01

[2] V. Dudnikov, Rev. Sci. Instr., 67(3), 915 (1996).

[3] S. Guharay et al, J.Vac.Sci.Tech.,B14(6),3907(1996).

[4] A.Bashkeev, V.Dudnikov, AIP Conf. No 210, p.329, 1990.

[5] C.W.Schmidt, V. Dudnikov, J. MacLachlan, PAC01, 2001.

[6] C. W. Schmidt, C. Curtis, IEEE Trans. Nucl. Sci., NS-26,4120(1979).

[7] K. Volk, PhD Thesis, University of Frankfurt, 1993.

[8] Jack Boers, Thunderbird Simulations, Garfield, TX. 